\title{
Ambiente comunitario y actitud hacia la autoridad: relaciones con la calidad de las relaciones familiares y con la agresión hacia los iguales en adolescentes
}

\author{
Teresa I. Jiménez ${ }^{1 *}$, Estefanía Estévez ${ }^{2}$ y Sergio Murgui ${ }^{3}$ \\ 1 Universidad de Zaragoza \\ 2 Universidad Miguel Hernánder \\ 3 Universidad de Valencia
} Resumen: El objetivo del presente estudio es explorar las relaciones entre
la calidad de las relaciones familiares, la percepción del ambiente comunita-
rio y la actitud de los adolescentes hacia la autoridad (policía, profesores) y
normas sociales, en la predicción del bienestar subjetivo y un problema
conductual de actualidad como es la agresión hacia los iguales. Para poner a
prueba estas relaciones múltiples se ha utilizado una muestra de 554 adoles-
centes de ambos sexos con edades comprendidas entre los 12 y los 16 años.
Los datos se recogieron en dos tiempos con un intervalo temporal de 6 me-
ses y se analizaron mediante el cálculo de modelos de ecuaciones estructu-
rales, incluyendo un análisis multigrupo por sexo. Los resultados obtenidos
indican que unas relaciones familiares positivas se relacionan con una per-
cepción positiva del ambiente comunitario y actitudes también positivas ha-
cia la autoridad y las normas sociales. Estos factores actúan como una pro-
tección frente a la posterior implicación en conductas agresivas hacia los
iguales en el contexto escolar y favorecen el bienestar subjetivo. El modelo
resultante es equivalente para chicos y chicas.
Palabras clave: Relaciones familiares; comunidad; actitud hacia la autori-
dad; bienestar subjetivo; agresión escolar. dad; bienestar subjetivo; agresión escolar.
Title: Community environment and attitude toward authority: relations with quality of family relationships and aggression toward peers in adolescents.

Abstract: The present study explores the relations between the perception of the family relationships, community environment and adolescents' attitude towards figures of authority (police and teachers) and social rules, in the prediction of subjective well-being and a current behavior problem like peer aggression. In order to test these multiple relations, we used a sample of 554 adolescents of both sexes, aged between 12 and 16 years. Data were collected in two waves with a 6-month interval and were analyzed by means of structural equation models, including a multigroup analysis by sex. The results obtained indicate that positive family relations are related to a positive perception of the community environment and also to positive attitudes towards authority and towards social rules. These factors act as protectors against subsequent involvement in aggressive behaviors towards peers in the school setting and they also promote subjective wellbeing. The resulting model is equivalent for boys and girls.

Key words: Family relationships; community; attitude towards authority; subjective well-being; school aggression.

\section{Introducción}

Desde un punto de vista epidemiológico, la agresión entre iguales es un hecho frecuente en los centros educativos y así ha sido constatado en numerosos estudios realizados con población escolar europea (Blaya, Debardieux, del Rey y Ortega, 2006; Del Barrio et al., 2008; Klicpera y GasteigerKlicpera, 1996; Olweus, 2001). Se ha considerado que la agresión entre compañeros es la forma más común de violencia escolar (Nansel et al., 2001). En un reciente estudio realizado en 38 países y regiones europeos se ha estimado una media de $38 \%$ de escolares victimizadas por sus compañeros (World Health Organization, 2012). Si bien no hay acuerdo entre los estudios realizados en cuanto a los índices de prevalencia ni a los indicadores de gravedad, sí que hay acuerdo en considerar la agresión entre compañeros como un obstáculo importante para el proceso de enseñanzaaprendizaje en el aula, así como para las relaciones sociales existentes en la misma, y que contribuye al desarrollo de importantes problemas psicológicos y de integración social tanto en los agresores como en las víctimas. Es por esto que un tópico relevante de investigación actual es el análisis de los factores que ponen en riesgo o protegen a los adolescentes de implicarse en conductas agresivas en el contexto escolar.

Desde una perspectiva ecosistémica, el modelo del Desarrollo Social de Hawkins, Catalano y Miller (1992) y la teoría

* Dirección para correspondencia [Correspondence address]:

Teresa I. Jiménez. Universidad de Zaragoza. Departamento de Psicología

y Sociología. Facultad de Ciencias Sociales y Humanas. Ciudad Escolar, s/n. 44003, Teruel (España). E-mail: tijimgut@unizar.es de la Conducta Problema de Jessor (1993), ofrecen un sugerente marco de análisis de los contextos del desarrollo adolescente que permiten explicar por qué algunos adolescentes presentan un especial riesgo para desarrollar problemas conductuales y por qué otros no. Hawkins y colaboradores (1992) consideran que diferentes factores de riesgo presentes en los distintos contextos sociales relevantes en la adolescencia (escuela, familia, iguales y comunidad) configuran una matriz biopsicosocial donde todos están relacionados e influyen conjuntamente en el funcionamiento del adolescente en diversos ámbitos. Jessor (1993) también destaca el concepto de interrelación como un elemento central, tanto para explicar el tipo de relación que mantienen entre sí los distintos contextos sociales, como para reconocer la interacción que se produce entre distintas conductas y factores más y menos saludables. En su modelo, Jessor sistematiza los factores que pueden influir en los problemas de los adolescentes en tres dominios: individual (por ejemplo, factores genéticos), conductual (por ejemplo, la asistencia o no a la escuela) y social. En el área social, Jessor diferencia el ámbito social, que alude a elementos estructurales como por ejemplo la pobreza o el desempleo, del ambiente percibido, que alude a factores como la calidad de las relaciones familiares o el apoyo percibido de padres, amigos y otros agentes de la comunidad.

La investigación empírica ha aportado numerosas evidencias que indican la presencia de múltiples factores de riesgo y de protección en estos contextos del desarrollo adolescente y que se asocian a los diferentes problemas típicamente adolescentes como son la sintomatología depresiva, la 
conducta delictiva y violenta o el consumo de sustancias (McGee, Williams, Poulton y Moffitt, 2000). Respecto del ámbito familiar, numerosos estudios han destacado que un contexto familiar negativo, caracterizado por conflictos frecuentes y bajos niveles de comunicación abierta, afecto y apoyo, es un factor de riesgo relevante para la presencia de distintos indicadores de desajuste psicológico y conductual en los hijos adolescentes. Los mismos resultados se han observado en el sentido inverso, constituyendo también la calidad de las relaciones familiares un contexto relevante de protección frente a estas problemáticas (para una revisión ver Parke, 2004). Específicamente, en relación con la agresión en el contexto escolar, el estilo permisivo o, al contrario, la utilización de una disciplina coercitiva, la tolerancia hacia la agresividad de los hijos y el conflicto familiar se han relacionado con la conducta agresiva hacia los iguales (Cava, Musitu y Murgui, 2006; Jiménez y Lehalle, 2012; Olweus, 1994). Además, es interesante destacar que, desde un punto de vista ecológico, muchos autores han resaltado que el funcionamiento positivo en el contexto familiar, además de constituir un factor de protección en sí mismo, es un importante predictor del buen funcionamiento del adolescente en otros contextos como el escolar y el comunitario (Bronfenbrenner, 1995; Trickett, 2009). Por ejemplo, en un reciente metanálisis realizado a partir de 11,741 artículos relacionados con la conducta parental y la agresión/victimización entre iguales se encuentra una influencia parental moderada sobre la conducta de agresión hacia los iguales en la escuela (Lereya, Samara y Wolke, 2013).

En relación con el contexto escolar, se ha destacado la importancia de la escuela como primera institución formal con la que el niño toma contacto en su desarrollo social, y el rol desempeñado por las actitudes que el adolescente desarrolla hacia esta institución, las normas sociales y las figuras de autoridad como un importante predictor de problemas de conducta agresiva. En efecto, los trabajos llevados a cabo en Reino Unido por Emler y Reicher indican que los adolescentes que presentan actitudes negativas hacia la institución escolar, las normas sociales asociadas a ésta y las figuras de autoridad como profesores y policía presentan más problemas de conducta delictiva y agresiva (Emler y Reicher, 1995). Según Emler (2009) la conducta agresiva es una medida de autoprotección del adolescente que no confía en la protección de las figuras de autoridad y en la eficacia de las normas sociales. Por el contrario, siguiendo la argumentación de este autor, los adolescentes que confían en la protección de las figuras de autoridad y en la eficacia de las normas sociales no tendrían necesidad de implicarse en dinámicas de tipo agresivo.

Por lo que respecta al ámbito comunitario, numerosos trabajos han relacionado sistemáticamente variables comunitarias con el ajuste psicosocial de los adolescentes. Existe abundante literatura que apunta que distintas variables comunitarias (por ejemplo, la desorganización social, los problemas en el vecindario, la participación en actividades del barrio, la integración comunitaria o la calidad de la interacción con el vecindario) se relacionan con medidas de salud mental y bienestar social y psicosocial (Albanesi, Cicognani y Zani, 2007; Felton y Shinn, 1992; Herrero y Gracia, 2007) e inversamente, también con indicadores de conducta problemática, tales como delincuencia y violencia (Ingoldsby et al., 2006; Mrug y Windle, 2009; Vieno, Nation, Perkins y Santinello, 2007). Es necesario remarcar que, tal y como señalan Hull, Kilbourne, Reece y Husaini (2008), en la literatura comunitaria se han privilegiado los análisis de variables comunitarias de tipo estructural (por ejemplo, pobreza, indicadores de desempleo o disponibilidad de servicios) y, cuando se estudia la conducta delictiva y violenta, se han utilizando generalmente muestras masculinas de población adulta o en la adolescencia tardía. Consideramos por tanto que se ha subestimado la importancia que tiene la comunidad para la vida de los adolescentes más jóvenes y sus potenciales beneficios para el ajuste conductual de chicos y chicas. En el presente trabajo nos interesa analizar el ambiente comunitario tal y como es percibido por el adolescente, en el sentido expresado por Jessor (1993), y en relación con un problema de conducta específico como es la agresión hacia los iguales en el contexto escolar.

Si bien los modelos señalados anteriormente (Bronfenbrenner, 1995; Hawkins et al., 1992; Jessor, 1993) apoyan la interrelación entre contextos para la comprensión de las conductas problemáticas en la adolescencia, es escasa la investigación que ilustra empíricamente las múltiples relaciones entre familia, escuela y comunidad en la predicción de problemas de conducta específicos. La influencia conjunta del ambiente comunitario percibido, la calidad de las relaciones familiares y las actitudes hacia figuras de autoridad y normas sociales en la agresión hacia los iguales ha sido poco explorada. Es posible que el análisis conjunto de estos factores pueda contribuir a la comprensión, a medio plazo, de esta forma particular de violencia escolar. En consonancia, siguiendo la citada perspectiva ecosistémica en este estudio se utiliza una prueba aplazada en el tiempo para conocer el impacto de las variables contextuales en variables resultado como el bienestar subjetivo y la participación en agresiones hacia los iguales. Específicamente se plantea la hipótesis de que, inicialmente, unas relaciones positivas en el contexto familiar se relacionarán de forma significativa con la percepción de un ambiente comunitario más positivo y con actitudes igualmente positivas hacia las normas y figuras de autoridad comunitarias (tiempo 1). En segundo lugar, este conjunto de variables interactuarán como un factor de protección que se relacionará con una menor implicación en conductas de agresión hacia los iguales y un mayor bienestar subjetivo pasados seis meses (tiempo 2). Esta hipótesis está representada en el modelo teórico recogido en la Figura 1. Finalmente, debido a la constatación en estudios previos de diferencias significativas entre sexos en agresión, obteniendo los chicos mayores puntuaciones en agresión directa o manifiesta (Andreou, 2000; Nansel et al., 2001; Olweus, 1997), se examinará si las relaciones entre las variables mencionadas pueden contribuir a explicar la agresión manifiesta en ambos sexos. 


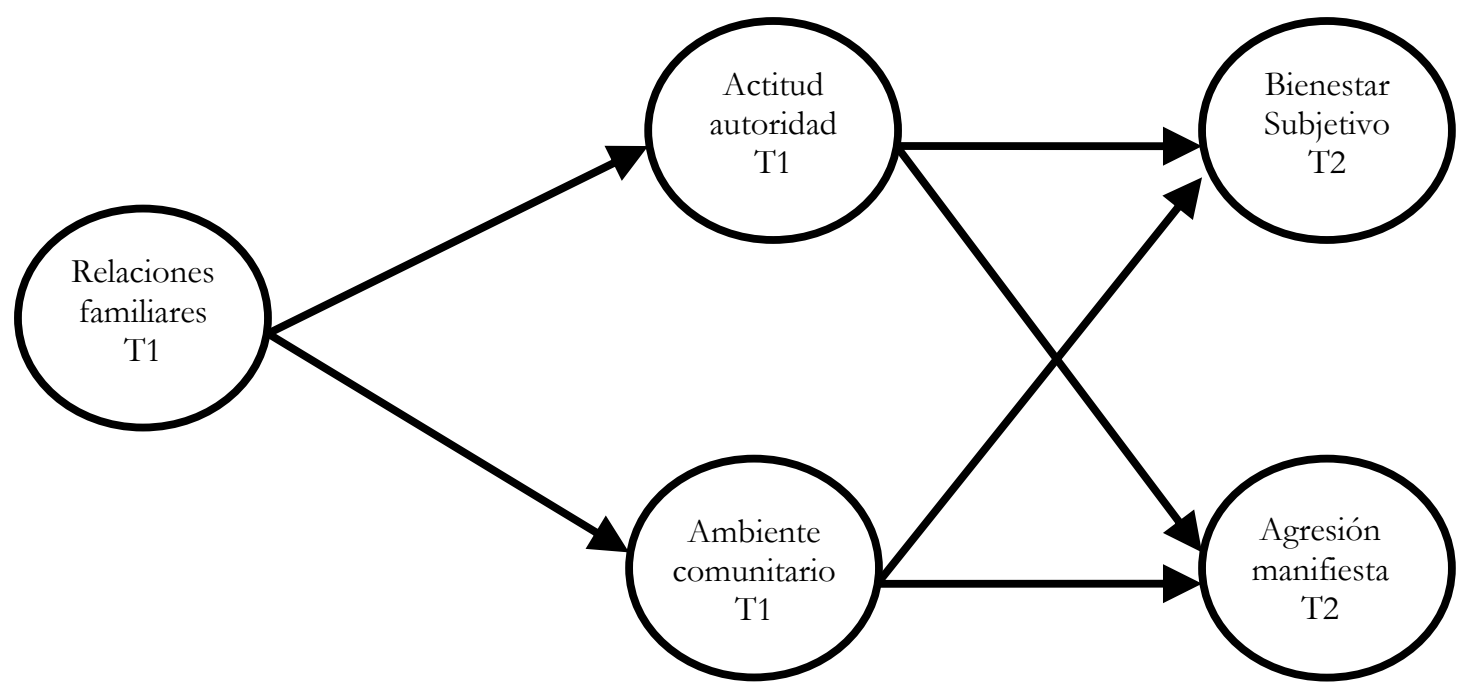

Figura 1. Modelo teórico.

\section{Método}

\section{Participantes}

La selección de los participantes se realizó mediante un muestreo estratificado por conglomerados (Santos, Muñoz, Juez y Cortiñas, 2003). Las unidades de muestreo fueron los centros educativos, públicos y concertados de entornos rurales y urbanos de colegios e institutos de la Comunidad Autónoma de Valencia. Los estratos se establecieron en función de la variable curso $\left(1^{\circ}, 2^{\circ}, 3^{\circ}\right.$ y $4^{\circ}$ de Educación Secundaria Obligatoria). Se asumió un error muestral de $\pm 2,3 \%$, un nivel de confianza del $95 \%$ y una varianza poblacional de .50. En el primer tiempo del estudio participaron 1319 adolescentes escolarizados en 7 centros públicos de Enseñanza Secundaria Obligatoria de la Comunidad Valenciana. En el segundo momento del estudio, 6 meses más tarde, se recuperó una muestra de 554 participantes debido a que 3 de los centros no volvieron a participar por cuestiones de organización horaria. La muestra final de este estudio se corresponde con los 554 adolescentes que participaron tanto en el primero como en el segundo tiempo. Los participantes tenían entre 12 y 16 años (edad media 13.5, desviación típica 1.6), 257 eran chicos $(46 \%)$ y 297 eran chicas (54\%). Se comprobó que las muestras fueran equivalentes en los dos momentos temporales: la media de edades fue similar en los dos grupos, con una diferencia de 0.2 años que, si bien es pequeña resultó significativa $\left(t_{1317}=2.92 ; p<.01\right)$, la proporción de chicos y chicas fue la misma en T1 y T2 $\left(\chi^{2}{ }_{1,1319}=2.376 ; p>.05\right)$. Tampoco se encontraron diferencias en el curso de los dos grupos, la proporción de $1^{\circ}, 2^{\circ}, 3^{\circ}$ y $4^{\circ}$ de la ESO fue similar en el grupo T1 y en el grupo del T2 $\left(\chi^{2}{ }_{3,1319}=5.691 ; p>\right.$ $.05)$.

\section{Procedimiento}

En primer lugar, se contactó con los centros educativos a través de sus directores y se realizó un seminario informativo con la plantilla de profesorado para explicar los objetivos, importancia y alcance del estudio. Seguidamente, se solicitó el consentimiento de los padres y madres mediante una carta explicativa de la investigación. En tercer lugar, se acordaron fechas para realizar la primera aplicación de los instrumentos con los alumnos. Esta tuvo lugar en sus aulas habituales, durante un período regular de clase de aproximadamente 45 minutos de duración y previa explicación de los aspectos más relevantes del estudio por parte de un miembro del equipo de investigación. En todos los casos, la participación de los adolescentes fue voluntaria y anónima. El mismo procedimiento se siguió para la realización de la segunda aplicación de los instrumentos seis meses más tarde.

\section{Instrumentos}

Relaciones familiares. Se utilizó la Escala de Comunicación Familiar de Barnes y Olson (1982) (versión en español de Equipo Lisis) que es un adecuado indicador de la calidad de las relaciones familiares (Barnes y Olson, 1985). Esta escala, en su vertiente de comunicación positiva, se compone de 10 ítems y dos sub-escalas que evalúan, con una escala de respuesta de 1 (muy en desacuerdo) a 4 (muy de acuerdo) la Comunicación Abierta (comunicación positiva, libre, comprensiva y satisfactoria) con la madre y con el padre (por ejemplo, "Me presta atención cuando le hablo"; "Puedo expresarle mis verdaderos sentimientos"). En estudios previos con otras muestras de adolescentes españoles se han obtenido índices aceptables de fiabilidad (alpha de Cronbach) de entre .64 y .91 (Jiménez, Estévez, Musitu y Murgui, 2007; Jiménez, Musitu, Ramos y Murgui, 2009). En el presente estudio, el coeficiente alpha de Cronbach es de .88 . 
Ambiente comunitario percibido. Se utilizó la Escala de Apoyo Social Comunitario percibido (PCSQ; Herrero y Gracia, 2007). Esta escala consta de 24 ítems que evalúan, con un rango de respuesta de 1 (nunca) a 4 (siempre) el ambiente comunitario percibido a través de cuatro dimensiones: Integración Comunitaria (grado de sentimiento de pertenencia y de identificación con la comunidad o vecindario; por ejemplo, "Siento el barrio como algo mío"), Participación Comunitaria (grado en que el adolescente se implica en actividades sociales en su comunidad; por ejemplo, "Colaboro -solo, con mi familia, con amigos...- en asociaciones o en actividades que se llevan a cabo en mi barrio"), Apoyo Social Informal (percepción del grado de disponibilidad de ayuda en personas de la comunidad; por ejemplo. "En mi barrio puedo encontrar personas que me ayudan a sentirme feliz") y Apoyo Social Formal (percepción del grado de disponibilidad de ayuda y de la confianza en organizaciones y servicios de la comunidad; por ejemplo, "En estos servicios encontraría ayuda si tuviera problemas personales, familiares, etc.”). En estudios previos, se ha mostrado que el PCSQ evalúa adecuadamente la experiencia comunitaria en jóvenes adultos y adolescentes, y se han encontrado coeficientes de fiabilidad (alpha de Cronbach) de entre .75 y .88 (Gracia, Herrero y Musitu, 2002, Herrero y Gracia, 2007). En nuestro estudio, el coeficiente alpha de Cronbach para estas dimensiones es de .69, $.68, .84$ y .68 respectivamente.

Actitud hacia la autoridad. Se ha elaborado una escala a partir de las escalas previas de Reicher y Emler (1985) y Rubini y Palmonari (1995). Ambas escalas se han utilizado en estudios previos, mostrando en todos los casos adecuadas propiedades psicométricas (Emler y Reicher, 1995, 2005; Rubini y Palmonari, 1998). El instrumento elaborado consta de 9 ítems, con una escala de 1 (muy en desacuerdo) a 4 (muy de acuerdo). Estos ítems recogen información sobre la actitud hacia determinadas figuras e instituciones de autoridad formal, como son la escuela y el profesorado, la policía y las leyes y normas socialmente establecidas. El análisis factorial de la escala mostró la existencia de dos factores, que explican en conjunto el $49.86 \%$ de la varianza total. El primer factor aglutina 5 ítems (33.84\% de la varianza explicada) que hacen referencia a la Actitud Positiva hacia la Autoridad (por ejemplo, "La policía está para hacer una sociedad mejor para todos"; "Los profesores son justos a la hora de evaluar"). El segundo factor agrupa 4 ítems $(16.02 \%$ de la varianza explicada) que reflejan una Actitud Positiva hacia la Transgresión de las Normas (por ejemplo, "Es normal desobedecer a los profesores si no hay castigos", "Es normal saltarse la ley si no se causa daño a nadie"). Para la realización de los análisis se invirtieron las puntuaciones de esta variable quedando denominada Actitud de No Transgresión de Normas. Los coeficientes de fiabilidad (alpha de Cronbach) para estas dimensiones en el presente estudio son de .76 y .67 respectivamente.

Agresión hacia los iguales. Se ha utilizado la Escala de Conducta Agresiva Manifiesta de Little, Henrich, Jones y Hawley (2003) (adaptación al español de Equipo Lisis). Esta escala consta de 13 ítems que evalúan, con un rango de respuesta de 1 (nunca) a 4 (siempre), la Conducta Agresiva Manifiesta (también denominada agresión directa) en el contexto escolar en sus formas pura (por ejemplo, "Soy una persona que se pelea con los demás"), reactiva (por ejemplo, "Cuando alguien me hace daño o me hiere, le pego") e instrumental (por ejemplo, "Amenazo a otros para conseguir lo que quiero). En estudios previos realizados con otras muestras de adolescentes españoles se han obtenido adecuadas propiedades psicométricas con índices de fiabilidad que oscilan entre .62 y .84 (Cava et al., 2006; Jiménez, Moreno, Murgui y Musitu, 2008; Musitu, Estévez y Emler, 2007). El alpha de Cronbach de las tres dimensiones en el presente estudio es $.72, .79$ y .87 respectivamente.

Bienestar subjetivo. Se ha utilizado la Escala de Satisfacción con la Vida de Diener, Emmons, Larsen, y Griffin (1985) (adaptación al español de Atienza, Pons, Balaguer y GarcíaMerita, 2000). Esta escala utiliza un rango de respuesta de 1 (muy en desacuerdo) a 4 (muy de acuerdo) y consta de 5 ítems (por ejemplo, "Mi vida es en la mayoría de los aspectos como me gustaría que fuera") que aportan un índice general de satisfacción con la vida, entendida ésta como un constructo general de Bienestar Subjetivo (Diener, Suh, Lucas y Smith, 1999). La consistencia interna de este instrumento en su versión original (alpha de Cronbach $=.84$ ) se ha confirmado en varias muestras españolas (Buelga, Musitu, Murgui y Pons, 2008; Chico, 2006; Extremera, Durán y Rey, 2007). En la presente muestra el alfa de Cronbach es de .77.

\section{Resultados}

Como un análisis exploratorio de las relaciones entre las dimensiones evaluadas, se calcularon correlaciones de Pearson entre todas las variables objeto de estudio y MANOVAS en función del sexo. En la Tabla 1 se presentan las correlaciones de Pearson, la media y la desviación típica de todas las variables. 
Tabla 1. Correlaciones de Pearson y estadísticos descriptivos de las variables observables.

\begin{tabular}{|c|c|c|c|c|c|c|c|c|c|c|c|c|c|}
\hline & & 1 & 2 & 3 & 4 & 5 & 6 & 7 & 8 & 9 & 10 & 11 & 12 \\
\hline 1 & $\begin{array}{l}\text { Comunicación } \\
\text { abierta con la } \\
\text { madre T1 }\end{array}$ & 1 & & & & & & & & & & & \\
\hline 2 & $\begin{array}{l}\text { Comunicación } \\
\text { abierta con el } \\
\text { padre T1 }\end{array}$ & $.605^{* * *}$ & 1 & & & & & & & & & & \\
\hline 3 & $\begin{array}{l}\text { Actitud positiva } \\
\text { hacia autoridad } \mathrm{T} 1\end{array}$ & $.195^{* * *}$ & $.173^{* * *}$ & 1 & & & & & & & & & \\
\hline 4 & $\begin{array}{l}\text { Actitud no } \\
\text { transgresión de } \\
\text { normas T1 }\end{array}$ & $.240^{* * *}$ & $.291 * * *$ & $.350 * * *$ & 1 & & & & & & & & \\
\hline 5 & $\begin{array}{l}\text { Integración } \\
\text { comunitaria T1 }\end{array}$ & $.096^{*}$ & $.128^{* *}$ & -.008 & .069 & 1 & & & & & & & \\
\hline 6 & $\begin{array}{l}\text { Participación } \\
\text { comunitaria T1 }\end{array}$ & $.097^{*}$ & $.123 * *$ & $.099 *$ & $.133^{* *}$ & $.351 * * *$ & 1 & & & & & & \\
\hline 7 & $\begin{array}{l}\text { Apoyo social } \\
\text { informal T1 }\end{array}$ & .060 & $.114 * *$ & .042 & $.084^{*}$ & $.541^{* * *}$ & $.338^{* * *}$ & 1 & & & & & \\
\hline 8 & $\begin{array}{l}\text { Apoyo social } \\
\text { formal T1 }\end{array}$ & $.117^{* *}$ & $.134^{* *}$ & $.130^{* *}$ & $.255^{* * *}$ & $.262^{* * *}$ & $.290^{* * *}$ & $.341^{* * *}$ & 1 & & & & \\
\hline 9 & $\begin{array}{l}\text { Satisfacción con } \\
\text { la vida T2 }\end{array}$ & $.283^{* * *}$ & $.241 * * *$ & $.093^{*}$ & $.182^{* * *}$ & $.246^{* * *}$ & $.099 *$ & $.181^{* * *}$ & $.217^{* * *}$ & 1 & & & \\
\hline 10 & Agresión pura T2 & $-.165^{* * *}$ & $-.149 * * *$ & $-.272^{* * *}$ & $-.282^{* * *}$ & $-.111 * *$ & $-.161 * * *$ & $-.116^{* *}$ & $-.208^{* * *}$ & $-.227 * * *$ & 1 & & \\
\hline 11 & Agresión reactivaT2 & $-.203 * * *$ & $-.190 * * *$ & $-.281 * * *$ & $-.294 * * *$ & -.024 & $-.096^{*}$ & -.059 & $-.135^{* *}$ & $-.187^{* * *}$ & $.596 * * *$ & 1 & \\
\hline 12 & $\begin{array}{l}\text { Agresión instru- } \\
\text { mental T2 }\end{array}$ & $-.173^{* * *}$ & $-.144^{* *}$ & $-.321 * * *$ & $-.251 * * *$ & $-.093^{*}$ & $-.103^{*}$ & $-.098^{*}$ & $-.157 * * *$ & $-.197^{* * *}$ & $.696^{* * *}$ & $.640 * * *$ & 1 \\
\hline & $\begin{array}{l}\text { Media chicos/ } \\
\text { chicas }\end{array}$ & $\begin{array}{c}41.06 / \\
42.40\end{array}$ & $\begin{array}{c}38.88 / \\
38.29\end{array}$ & $\begin{array}{l}7.42 / \\
16.83\end{array}$ & $\begin{array}{c}15.74 / \\
16.04\end{array}$ & $\begin{array}{c}15.31 / \\
16.00\end{array}$ & $\begin{array}{l}16.61 / \\
16.67\end{array}$ & $\begin{array}{l}24.46 / \\
26.05\end{array}$ & $\begin{array}{c}10.42 / \\
10.78\end{array}$ & $\begin{array}{c}14.67 / \\
14.59\end{array}$ & $\begin{array}{c}6.76 / \\
5.88\end{array}$ & $\begin{array}{l}8.06 / \\
6.28\end{array}$ & $\begin{array}{r}8.07 / \\
6.59\end{array}$ \\
\hline & $\begin{array}{l}\text { Desviación típica } \\
\text { chicos/chicas }\end{array}$ & $\begin{array}{r}8.32 / \\
8.85\end{array}$ & $\begin{array}{c}8.77 / \\
9.35\end{array}$ & $\begin{array}{r}2.85 / \\
2.92 \\
\end{array}$ & $\begin{array}{c}3.79 / \\
3.81\end{array}$ & $\begin{array}{r}2.85 / \\
2.97\end{array}$ & $\begin{array}{r}4.62 / \\
4.87\end{array}$ & $\begin{array}{c}5.58 / \\
5.78\end{array}$ & $\begin{array}{r}2.79 / \\
2.74\end{array}$ & $\begin{array}{r}2.98 / \\
3.17\end{array}$ & $\begin{array}{c}2.25 / \\
1.67\end{array}$ & $\begin{array}{r}2.73 / \\
2.09 \\
\end{array}$ & $\begin{array}{r}3.24 / \\
2.20 \\
\end{array}$ \\
\hline
\end{tabular}

Notas: las variables están estandarizadas; T1 = variable evaluada en tiempo 1, T2 = variable evaluada en tiempo 2 ${ }^{*} p<.05 ; * * p<.01 ; * * * p<.001$

La mayoría de las correlaciones fueron significativas y en la dirección esperada. Destacan, en un sentido positivo, las correlaciones altas entre la comunicación abierta con ambos padres y actitudes positivas hacia la autoridad y la no transgresión de normas sociales y, en un sentido negativo, las correlaciones entre estas actitudes y una menor implicación en agresión hacia los iguales. La integración comunitaria y el apoyo social informal no correlacionaron con las dimensiones actitudinales y con la agresión reactiva. Se realizó un MANOVA en función del sexo que no mostró diferencias en los factores de ambiente comunitario percibido (Wilks $=$ $.984 ; F(4,421)=1.757 ; p>.05)$, actitud hacia la autoridad institucional y no transgresión de normas sociales (Wilks = .989; $F(2,551)=2,930 ; p>$.05). Sí se encontraron diferencias entre sexos en comunicación familiar (Wilks $=.983 ; F$ $(2,464)=1.757 ; p<.05)$, si bien las diferencias no resultaron significativas en los ANOVAS correspondientes $(F(1,468)$ $=2.929$ para comunicación abierta con el padre y $F(1,468)$ $=0.569$ para comunicación abierta con la madre, ambas $p>$ $.05)$. La prueba $F$ para satisfacción con la vida tampoco resultó significativa $(F(1,552)=.083 ; p>.05)$. Sin embargo, sí que se observaron diferencias entre sexos en violencia manifiesta (Wilks $=.875 ; F(3,550)=26.138 ; p<.01)$, en sus tres dimensiones: pura $(F(1,552)=27.51 ; p<.01)$, reactiva $(F(1,552)=76.178 ; p<.01)$ e instrumental $(F(1,468)=$
40.601; $p<.01$ ), siendo los chicos los que presentaron mayores puntuaciones.

Seguidamente, se utilizó el programa EQS 6.0 (Bentler, 1995) para calcular un modelo de ecuaciones estructurales que tuviese en cuenta las múltiples relaciones entre todas las variables del estudio tal y como se había planteado en la hipótesis de partida. El modelo incluyó 5 factores latentes estimados a partir de las variables observables tal y como se describe a continuación: Relaciones Familiares, compuesto de las dimensiones comunicación abierta con la madre y comunicación abierta con el padre; Actitud hacia la Autoridad, compuesto de las variables actitud positiva hacia la autoridad y no transgresión de normas sociales; Ambiente Comunitario Percibido, calculado a partir de las dimensiones de participación comunitaria, integración comunitaria, apoyo social informal y apoyo social formal; Agresión hacia Iguales, conformado por las variables de agresión pura, agresión reactiva y agresión instrumental; y Bienestar Subjetivo, formado por un único indicador de satisfacción con la vida y, por tanto, con saturación factorial de 1 y error igual a 0 . En la tabla 2 se presentan las saturaciones de cada indicador en el factor latente correspondiente y los errores asociados. 
Tabla 2. Saturaciones factoriales, errores estándar y probabilidad asociada

\begin{tabular}{ll}
\hline Factores y variables & Cargas factoriales \\
\hline Relaciones familiares & \\
Comunicación positiva padre & $.926^{* * *}(.127)$ \\
$\quad$ Comunicación positiva madre & $1^{\text {a }}$ \\
Actitud hacia la autoridad & \\
$\quad$ Actitud positiva figuras autoridad & $1^{\text {a }}$ \\
$\quad$ No transgresión normas & $.987^{* * *}(.166)$ \\
Ambiente Comunitario & \\
$\quad$ Integración & $1^{\text {a }}$ \\
Participación & $.958^{* * *}(.034)$ \\
Apoyo informal & $1,044^{* * *}(.148)$ \\
Apoyo formal & $.691^{* * *}(.118)$ \\
Bienestar Subjetivo & $1^{\text {a }}$ \\
Agresión manifiesta & \\
Agresión pura & $1^{\text {a }}$ \\
Agresión reactiva & $.883^{* * *}(.068)$ \\
Agresión instrumental & $.958^{* * *}(.068)$ \\
\hline
\end{tabular}

a Fijados en 1.00 durante la estimación.

$* * * p<.001$

Para conocer la bondad de ajuste del modelo a los datos y la significación estadística de los coeficientes, se utilizaron estimadores robustos debido a la desviación de la normalidad de los datos (coeficiente mardia normalizado $=6.98$ ). Aunque, en principio, se incluyó en el modelo la correlación entre el factor de agresividad y el de bienestar subjetivo, lo que mejoraba el ajuste global del modelo $\left(\Delta \chi_{1}=-5.01 ; p<\right.$ $.05)$, dicha correlación no resultó significativa $(r=-.14 ; p>$ .05) por lo que se decidió no incluirla en el modelo final. El modelo final calculado ajustó bien a los datos, tal y como indican los siguientes índices: S-B $\chi^{2}(28, N=554)=65.65, p$ $<.05 ; C F I=.98, I F I=.98, N N F I=.96$, y RMSE $A=.038$ (90\%, .005-.059). Para los índices CFI, IFI y NNFI se consideran aceptables valores superiores a .95 , y para el índice RMSE $A$ valores inferiores a .05 (Batista y Coenders, 2000). Este modelo explicó el 17\% de la varianza del factor Bienestar Subjetivo y el 49\% del factor Agresión Manifiesta, lo que parece evitar un error de tipo II (García, Pascual, Frías, Van Krunckelsven y Murgui, 2008). La Figura 2 muestra la representación gráfica del modelo estructural final con los coeficientes estandarizados y su probabilidad asociada.

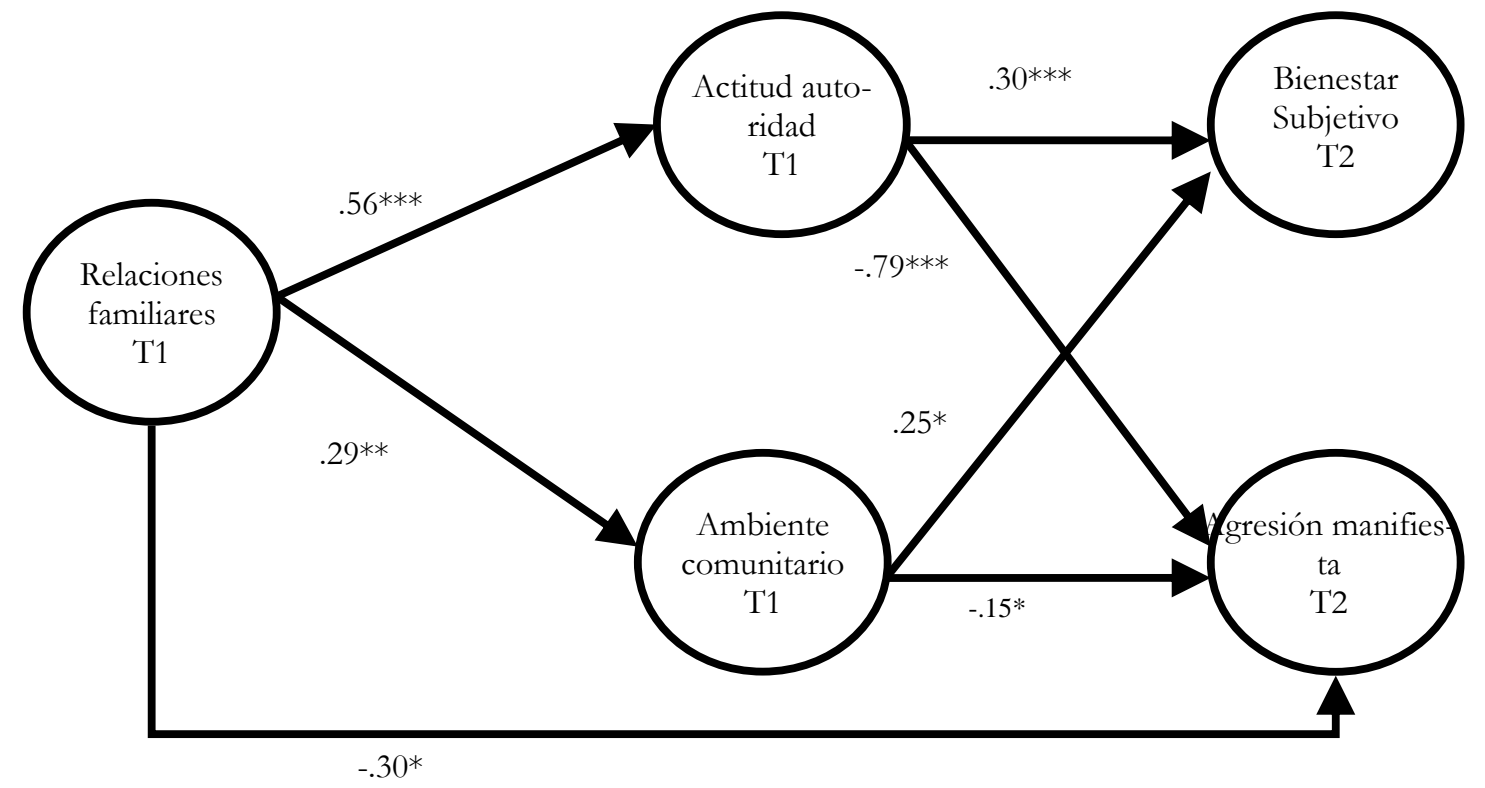

Figura 2. Modelo estructural final ${ }^{*} p<.05 ;{ }^{* *} p<.01 ;{ }^{* * *} p<.001$

En los resultados del modelo presentados en la figura 2 se observa una relación de protección tanto directa como indirecta entre las relaciones familiares y la posterior conducta agresiva del adolescente hacia sus iguales en la escuela. En el primer caso, observamos una relación significativa y negativa entre la percepción de unas relaciones positivas en el contexto familiar en el tiempo 1 y la implicación del adolescentes en conductas de tipo agresivo hacia sus iguales pasados 6 meses $(\beta=-.30 ; p<.05)$. En el segundo caso, estas relaciones familiares positivas se relacionan de forma significa- tiva y positiva, en el tiempo 1 , con una actitud positiva hacia la autoridad $(\beta=.56 ; p<.001)$ y la percepción positiva del ambiente comunitario $(\beta=.29 ; p<.01)$. A su vez, estos dos factores muestran una relación significativa con el bienestar subjetivo y con la conducta agresiva hacia los iguales pasados seis meses. Específicamente, tanto la actitud positiva hacia la autoridad como el ambiente comunitario percibido se relacionan de forma positiva con el posterior bienestar subjetivo $(\beta=.3 . p<.001 ; \mathrm{y} \beta=.25, p<.001$ respectivamente), mientras que lo hacen de forma negativa cuando se trata de la 
agresión hacia los iguales 6 meses después $(\beta=-.79, p<$ $.001 ;$ y $\beta=-.15, p<.05$ respectivamente). Estos resultados indican que las relaciones familiares positivas se relacionan con actitudes también más positivas hacia la autoridad y con la percepción de implicación y apoyo en la comunidad, un aspecto que a su vez, favorece el bienestar subjetivo y dificulta que el adolescente se implique en conductas de agresión hacia sus iguales en la escuela 6 meses después. Además, se observa una relación directa de protección de la calidad de las relaciones familiares frente al posterior desarrollo de conductas de agresión hacia los iguales.

Finalmente, con el objeto de contrastar si el modelo propuesto es válido para chicos y chicas, se realizó una comparación multigrupo (Bentler y Wu, 2002). Se calcularon dos modelos: el modelo sin restricciones, donde los parámetros se estimaron libremente, y el modelo restringido, en el que se consideran invariantes los parámetros del modelo en ambos grupos. Si la $\chi^{2}$ del modelo restringido fuera significativamente mayor que la $\chi^{2}$ del modelo sin restricciones, la hipótesis de invarianza no sería válida. Los resultados mostraron que el modelo restringido y no restringido son estadísticamente equivalentes entre ambos grupos para los paths y relaciones propuestos $\left(\Delta \chi^{2}(7, N=554)=5.38 ; p>.05\right)$. Por tanto, la invarianza factorial entre los dos grupos refuerza la generalización del modelo propuesto a ambos sexos.

\section{Discusión}

El presente estudio tenía como objetivo analizar la interrelación entre la calidad de las relaciones familiares, el ambiente comunitario percibido y las actitudes hacia la autoridad de los adolescentes en la predicción del bienestar subjetivo y la agresión manifiesta hacia los iguales en la escuela. Por un lado, los resultados han indicado que la conducta agresiva manifiesta hacia los iguales es la única variable del estudio que discrimina entre chicos y chicas, siendo los chicos los que se implican con mayor frecuencia en esta forma de violencia escolar. Nuestros resultados confirman los ya encontrados en numerosos estudios realizados con distintas muestras y en diferentes países (Andreou, 2000; Estévez, Martínez, Herrero y Musitu, 2006; Olweus, 1997). En estos estudios se ha observado que los chicos adolescentes se implican en mayor medida en conductas agresivas de tipo directo (tales como insultar, golpear, zarandear, empujar, etc.) que indirecto (rumorear sobre alguien o excluirlo de un grupo) sean estas conductas indiscriminadas, una reacción a una agresión previa percibida o un medio para conseguir algún beneficio.

Por otro lado, los resultados obtenidos añaden evidencia empírica a los modelos psicosociales y ecosistémicos del desarrollo de problemas de conducta en la adolescencia, específicamente en relación con la conducta agresiva hacia los iguales en la escuela. Tal y como sostienen Hawkins y colaboradores (1992) y Jessor (1993), factores procedentes de los distintos contextos importantes del desarrollo adolescente interactúan para dar lugar a un cómputo de riesgo y/o pro- tección asociado a los problemas conductuales. En relación con el contexto familiar, de acuerdo con estudios previos (Cava et al., 2006; Jiménez y Lehalle, 2012; Lereya et al., 2013; Olweus, 1994) se observa que las relaciones positivas entre padres e hijos, caracterizadas por una comunicación familiar positiva, libre, comprensiva y satisfactoria, constituyen un factor de protección directo de la agresión hacia los iguales en los hijos. Además, parece que las relaciones familiares positivas son un caldo de cultivo para el desarrollo de percepciones positivas en otros contextos y relaciones interpersonales del adolescente. La relación con los padres como primeras figuras de autoridad y fuente de apoyo para el adolescente parece constituir un modelo que puede estar influyendo en la percepción de otras figuras de autoridad extrafamiliares como la policía o los profesores y de otras relaciones de apoyo social como las comunitarias, tanto formales (servicios sociales comunitarios) como informales (vecinos y otros adultos).

Estos resultados también son coherentes con la perspectiva de la teoría del apego de Bowlby (1969). Según Bowlby, la calidad de las relaciones del niño en su familia configuran sus modelos cognitivos internos de representación del self y de las relaciones con los demás. Estos modelos influyen en la percepción acerca de la disponibilidad de los demás e influyen en su capacidad posterior para percibir apoyo y protección tanto de los padres como de otras personas significativas. Según estudios más recientes, dicha capacidad, aunque resistente al cambio, puede alterarse a lo largo del ciclo vital $y$, por lo tanto, se observa que la calidad de las relaciones entre padres-hijos durante la adolescencia es todavía una fuente importante de influencia en la percepción de los hijos de otros recursos de apoyo y protección extrafamiliares (Sarason et al., 1991; Simons, Chao, Conger y Elder, 2001).

Los resultados indican además que las actitudes positivas hacia la autoridad institucional (en la figura de profesores y policía) y hacia las normas sociales (escolares y legales) son un importante factor de protección frente a la conducta de agresión hacia los iguales y un factor promotor del bienestar subjetivo de los adolescentes a medio plazo. Estos resultados añaden consistencia a los obtenidos por otros autores (Emler y Reicher, 1995; Estévez, Murgui, Moreno y Musitu, 2007) y confirman la importancia de la actitud positiva hacia la autoridad institucional como un factor de protección directo frente a la implicación de chicos y chicas en conductas de agresión en la escuela. Según la perspectiva de Emler (2009), la actitud negativa hacia las figuras de autoridad y normas sociales se relaciona con la percepción de los adolescentes de que estas autoridades (policía y profesores) y normas son ineficaces en su protección frente a situaciones de conflicto por lo que los adolescentes recurrirían a la autodefensa mediante la agresión hacia otros iguales potencialmente agresores. Estudios previos han aportado evidencia en este sentido (Estévez, Jiménez y Moreno, 2010) y, en general, estos resultados refuerzan la necesidad de considerar en los programas de prevención de la violencia en las escuelas el desarrollo de actitudes positivas hacia la autoridad y las 
normas sociales como fuentes de seguridad, confianza y protección.

Respecto del papel del ambiente comunitario percibido, nuestros resultados también son consistentes con estudios previos que informan de los efectos beneficiosos del sentimiento de integración, implicación y apoyo en la propia comunidad para el ajuste psicosocial de los adolescentes (Albanesi et al., 2007; Herrero y Gracia, 2007; Vieno et al., 2007). En efecto, se observa que los adolescentes que se muestran en consonancia con las normas y figuras de autoridad de su comunidad, que se perciben integrados, participan en la misma e informan de elevados niveles de apoyo social formal (recursos comunitarios) e informal (vecinos y adultos en general), se benefician de una mayor satisfacción con sus vidas y de menores niveles de agresión hacia los iguales en la escuela. Estos resultados sugieren que no se debe subestimar la importancia del Sense of Community, en el sentido formulado por Mc Millan y Chavis (1986) -sentimiento de formar parte de una comunidad, oportunidades para participar en la vida comunitaria, integración y satisfacción de necesidades y conexión emocional compartida- en la prevención de un problema de actualidad como es la violencia escolar. Por tanto, consideramos que las acciones dirigidas a favorecer la participación de los adolescentes en organizaciones comunitarias (voluntariado, ocio, etc.), facilitar su sentimiento de integración en estas organizaciones y mejorar su confianza en las mismas, favorecerán, a su vez, la confianza en las relaciones interpersonales que pueden encontrar en su vecindario y su percepción de ayuda y protección tanto formal como informal de figuras de autoridad comunitarias como profesores y policía.

Finalmente, lo que nuestros resultados también parecen apuntar es que, independientemente del nivel de implicación en conductas de agresión manifiesta, el patrón de interrelación entre factores de protección analizado en este estudio es válido tanto para chicos como para chicas. En efecto, parece que tener unas buenas relaciones familiares, percibir el ambiente comunitario como un contexto de integración, participación y apoyo, y tener actitudes positivas hacia la autoridad institucional y las normas sociales, constituye un cómputo de protección que mejora el bienestar subjetivo y disminuye la probabilidad de agresión manifiesta hacia los iguales, tanto para los chicos como para las chicas. Este resultado es interesante puesto que permite argumentar el fomento de propuestas de intervención preventivas comunes para chicos y para chicas adolescentes, independientemente

\section{Referencias}

Albanesi, C., Cicognani, E. y Zani, B. (2007). Sense of community, civic engagement and social well-being in Italian adolescents. Journal of Community and Applied Psychology, 17, 387-406. Doi: 10.1002/casp.903

Andreou, E. (2000). Bully/Victim problems and their association with psychological constructs in 8-to 12-year old Greek schoolchildren. Aggressive Behavior, 26, 49-56. Doi: 10.1080/01443410020019830 de la cantidad de agresión directa que manifiestan hacia los compañeros.

El presente estudio también presenta ciertas limitaciones que es necesario señalar. En primer lugar, todos los datos recogidos en este estudio proceden de medidas de autoinforme. Este tipo de medidas son imprescindibles para obtener información de la percepción de los ambientes comunitario y familiar y de las actitudes de los adolescentes; sin embargo, pueden ser susceptibles de sesgos en las respuestas sobre agresión que pueden minar la validez y generabilidad de los resultados. En el caso de esta variable consideramos que sería necesario contrastar el autoinforme con un informe procedente del profesor tutor o psicopedagogo del centro educativo. En segundo lugar, las variables independientes analizadas en este estudio se refieren fundamentalmente a la evaluación de ambientes percibidos por el adolescente. Tal y como sugieren otros trabajos (Buelga, Cava y Musitu, 2012; Sánchez, Ortega y Menesini, 2012), variables de corte individual relacionadas con la autopercepción y la competencia emocional (por ejemplo, autoestima y regulación emocional) podría explicar y mediar la relación entre los contextos percibidos y las consecuencias conductuales y de bienestar subjetivo. Finalmente, en futuras investigaciones sería útil incluir medidas en un tercer tiempo que permitan identificar con mayor detalle el sentido de la influencia y el análisis del papel de estas terceras variables de corte individual en la explicación de las relaciones entre familia, comunidad y escuela y agresión hacia los iguales.

A pesar de estas limitaciones, el estudio ofrece elementos que ayudan a comprender cómo la agresión hacia los iguales en el contexto escolar se desarrolla y se expresa dentro de una constelación compleja de factores explicativos. Los esfuerzos para la prevención deberían orientarse hacia la aproximación entre familia, comunidad y centro educativo, creando, en palabras de Bronfenbrenner (1995), un mesosistema o ambiente de seguridad y desarrollo positivo mediante la coordinación de las figuras relevantes de apoyo y protección para los adolescentes como son padres, profesores, policía y vecindario.

Agradecimientos.- Este estudio ha sido elaborado en el marco del proyecto de investigación PSI2012-33464 "La violencia escolar, de pareja y filio-parental en la adolescencia desde la perspectiva ecológica", subvencionado por el Ministerio de Economía y Competitividad de España.

Atienza, F. L., Pons, D., Balaguer, I. y García-Merita, M. (2000). Propiedades psicométricas de la escala de satisfacción con la vida en adolescentes. Psicothema, 12, 314-320.

Barnes, H. y Olson D. H. (1982). Parent adolescent communication scale. En D. H. Olson, H. McCubbin, H. Barnes, A. Larsen, M. Muxen y W. Wilson (Eds.), Family Inventories: Inventories Used in a National Survey of Families across the Family Life Cycle (pp. 33-48). St. Paul: University of Minnesota Press. 
Barnes, H. y Olson, D. H. (1985). Parent-adolescent communication and the circumplex model. Child Development, 56, 438-447.

Batista, J. M. y Coenders, G. (2000). Modelos de ecuaciones estructurales. Madrid: La Muralla.

Bentler, P. M. (1995). EQS structural equations program manual. Encino, CA: Multivariate Software.

Bentler, P. y Wu, E. (2002). EQS 6 for windows user's guide. Encino, CA: Multivariate Software.

Blaya, C., Debardieux, E., del Rey, R. y Ortega, R. (2006). Clima y violencia escolar. Un estudio comparativo entre España y Francia. Revista de Educación, 339, 293-315.

Bowlby, J. (1969). Attachment and loss: Vol. 1. Attachment. New York: Basic Books.

Bronfenbrenner, U. (1995). The bio-ecological model from a life course perspective: reflections of a participant observer. En P. Moen, G. H. Elder, Jr. y K. Luscher, (Eds.), Examinig lives in context (pp. 599-618). Washington, DC: American Psychological Association.

Buelga, S., Cava, M. J. y Musitu, G. (2012). Reputación social, ajuste psicosocial y victimización entre adolescentes en el contexto escolar. Anales de Psicología, 28 (1), 180-187.

Buelga, S., Musitu, G., Murgui, S. y Pons, J. (2008). Reputation, loneliness, satisfaction with life and aggressive behavior in adolescence. The Spanish Journal of Psychology, 11 (1), 192-200.

Cava, M.J., Musitu, G. y Murgui, S. (2006). Familia y violencia escolar: el rol mediador de la autoestima y la actitud hacia la autoridad institucional. Psicothema, 18 (3), 367-373.

Chico, E. (2006). Personality dimensions and subjective well-being. The Spanish Journal of Psychology, 1 (9), 38-44.

Del Barrio, C., Martín, E., Montero, I., Gutiérrez, H., Barrios, A. y De Dios, M. (2008). Bullying and social exclusion in Spanish secondary schools: National trends from 1999 to 2006. International Journal of Clinical and Health Psychology, 8, 657-677.

Diener, E., Emmons, R., Larsen, R. J. y Griffin, S. (1985). The Satisfaction With Life Scale. Journal of Personalitiy Assessment, 49, 71-75. Doi: 10.1207/s15327752jpa4901_13

Diener, E., Suh, E. M., Lucas, R. E. y Smith, H. L. (1999). Subjective wellbeing: Three decades of progress. Psychological Bulletin, 125, 276-302. Doi: $10.1037 / / 0033-2909.125 .2 .276$

Emler, N. (2009). Delinquents as a minority group: Accidental tourists in forbidden territory or voluntary émigrés? En F. Butera y J. Levine (Eds.), Coping with minority status: Responses to exclusion and inclusion (pp. 127-154). US: Cambridge University Press.

Emler, N. y Reicher, S. (1995). Adolescence and delinquency. Oxford: Blackwell.

Emler, N. y Reicher, S. (2005). Delinquency: cause or consequence of social exclusion? In D. Abrams, J. Marques y M. Hogg (Eds), The social psychology of inclusion and exclusion (pp. 211-241). Philadelphia: Psychology Press.

Equipo Lisis. Escala de comunicación padres-adolescentes. Recuperado de http://www.uv.es/lisis/instrumentosnew.htm

Estévez, E., Jiménez, T. y Moreno, D. (2010). Cuando las víctimas de violencia escolar se convierten en agresores: “Quién va a defenderme?”. European Journal of Education and Psychology, 3 (2), 177-186.

Estévez, E., Martínez, B., Herrero, J. y Musitu, G. (2006). Aggressive and non-aggressive rejected students: an analysis of their differences. Psychology in the Schools, 43, 387-400.

Estévez, E., Murgui, S., Moreno, D. y Musitu, G. (2007). Estilos de comunicación familiar, actitud hacia la autoridad institucional y conducta violenta del adolescente en la escuela. Psicothema, 19, 108-113.

Extremera, N., Durán, A., y Rey, L. (2007). Perceived emotional intelligence and dispositional optimism-pessimism: Analyzing their role in predicting psychological adjustment among adolescents. Personaliy and Individual Differences, 42, 1069-1079.

Felton, B. y Shinn, M. (1992). Social integration and social support: Moving "social support" beyond the individual level. Journal of Community Psychology, 2. 103-115. Doi: 10.1002/1520-6629(199204)20:2<103::AIDJCOP2290200202>3.0.CO;2-4

García, J. F., Pascual, J., Frías, M. D., Van Krunckelsven, D. y Murgui, S. (2008). Diseño y análisis de la potencia: $n$ y los intervalos de confianza de las medias. Psicothema, 20 (4), 933-938.

Gracia, E., Herrero, J. y Musitu, G. (2002). Evaluación de recursos y estresores psicosociales en la comunidad. Madrid: Síntesis.
Hawkins, J. D., Catalano, R. F. y Miller, J. Y. (1992). Risk and protective factors for alcohol and other drug problems in adolescence and early adulthood: Implications for substance abuse prevention. Psychological Bulletin, 112, 64-105.

Herrero, J. y Gracia, E. (2007). Measuring perceived community support: factorial structure, longitudinal invariance, and predictive validity of the PCSQ (Perceived Community Support Questionnaire). Journal of Community Psychology, 35 (2), 197-217. Doi: 10.1002/jcop.20143

Hull, P., Kilbourne, B., Reece, M. y Husaini, B. (2008). Community involvement and adolescent mental health: moderating effects of race/ethnicity and neighborhood disadvantage. Journal of Community Psychology, 36 (4), 534-551. Doi: 10.1002/jcop.20253

Ingoldsby, E. M., Shaw, D. S., Winslow, E., Schonberg, M., Gilliom, M. y Criss, M. M. (2006). Neighborhood disadvantage, parent-child conflict, neighborhood peer relationships, and early antisocial behavior problem trajectories. Journal of Abnormal Child Psychology, 34, 293-309. Doi: 10.1007/s10802-006-9026-y

Jessor, R. (1993). Succesful adolescent development among youth in highrisk settings. American Psychology, 48, 117-126.

Jiménez, T. I., Estévez, E., Musitu, G. y Murgui, S. (2007). Comunicación familiar y comportamientos delictivos en la adolescencia: el doble rol mediador de la autoestima. Revista Latinoamericana de Psicología, 39 (1), 473- 485.

Jiménez, T.I. y Lehalle, H. (2012). La violencia escolar entre iguales en alumnos populares y rechazados. Psychosocial Intervention, 21 (1), 77-89. Doi: http://dx.doi.org/10.5093/in2012v21n1a5

Jiménez, T. I., Moreno, D., Murgui, S. y Musitu, G. (2008). Factores psicosociales relacionados con el estatus social del alumno en el aula: El rol de la reputación social, la amistad, la conducta violenta y la relación con el profesor. International Journal of Psychology and Psychological Therapy, 8 , 227-236.

Jiménez, T. I., Musitu, G., Ramos, M. J. y Murgui, S. (2009). Community involvement and victimization at school: an analysis through family, personal and social adjustment. The Journal of Community Psychology, 37 (8), 959-974. Doi: 10.1002/jcop.20342

Klicpera, A. C. y Gasteiger-Klicpera, B. G. (1996). Die Situation von 'Tätern' und 'Opferm' aggressiver Handlungen in der Schule, Praxis der Kinderpsychologie und inderpsychiatrie, 45, 2-9.

Lereya, S. T., Samara, M. y Wolke, D. (2013). Parenting behavior and the risk of becoming a bully/victim: a meta-analysis study. Child Abuse \& Neglect, 37 (12), 1091-1108.

Little, T. D., Henrich, C. C., Jones, S. M. y Hawley, P. H. (2003). Disentangling the "whys" from the "whats" of aggressive behaviour. International Journal of Behavioral Development, 27, 122-133. Doi: 10.1080/01650250244000128

McGee, R., Williams, S., Poulton, R. y Moffitt, T. (2000). A longitudinal study of cannabis use and mental health from adolescence to early adulthood. Addiction, 95 (4), 491-503. Doi: 10.1046/j.13600443.2000.9544912.x

Mc Millan, W. D. y Chavis, M. D. (1986). Sense of Community: A definition and a theory. Journal of Community Psychology, 14, 6-22.

Musitu, G., Estévez, E. y Emler, N. (2007). Adjustment problems in the family and school contexts, attitude towards authority and violent behaviour at school in adolescence. Adolescence, 42, 779-794.

Mrug, S. y Windle, M. (2009). Mediators od neigborhood influences on externalizing behavior in preadolescent children. Journal of Abnormal Cbild Psychology, 37, 265-280. Doi: 10.1007/s10802-008-9274-0.

Nansel, T. R., Overpeck, M., Pilla, R. S., Ruan, W. J., Simons-Morton, B. y Scheidt, P. (2001). Bullying behaviors among US youth: Prevalence and association with psychosocial adjustment. Journal of the American Medical Association, 285, 2094-2100.

Olweus, D. (1994). Annotation: Bullying at school: Basic facts and effects of a school based intervention program. Journal of Child Psychology and Psychiatry, 35, 1171-1190.

Olweus, D. (1997). Bully/victim problems in schools: Knowledge base and an effective intervention programme. Irish Journal of Psychology, 18, 170190.

Olweus, D. (2001). Peer harassment: A critical analysis and some important issues. In J. Juvonen y S. Graham (Eds.). Peer harassment in school: The plight of the vulnerable and victimized (pp. 3-20). New York: Guilford Press. 
Parke, R. D. (2004). Development in family. Annual Review of Psychology, 55 , 365-399.

Reicher, S. y Emler, N. (1985). Delinquent behavior and attitudes to formal authority. British Journal of Social Psychology, 3, 161-168.

Rubini, M. y Palmonari, A. (1995). Orientamenti verso la autorita formali e participazione politica degli adolescenti. Giornale Italiano di Psicologia, 10 (3), 757-775.

Rubini, M. y Palmonari, A. (1998). The effect of school maladajustment and of membership of adolescent peer-groups on the orientation to the institutional system. Comunicacion presentada a la 6th Biennal Conference of the EARA, Budapest.

Sánchez, V., Ortega, R. y Menesini, E. (2012). La competencia emocional de agresores y víctimas de bullying. Anales de Psicología, 28 (1), 71-82.

Santos, J., Muñoz, A., Juez, P. y Cortiñas, P. (2003). Diseño de encuestas de estudio de mercado. Técnicas de muestreo y análisis multivariante. Madrid: Fundación Ramón Areces.

Sarason, B. R., Pierce, G. R., Shearin, E. N., Sarason, I. G., Waltz, J. A. y Poppe, L. (1991). Perceived social support and working models of self and actual others. Journal of Personality and Social Psychology, 6. 273-287.
Simons, R. L., Chao, W., Conger, R. D. y Elder, G. H. (2001). Quality of parenting as mediator of the effect of childhood defiance on adolescent friendship choices and delinquency: A growth curve analysis. Journal of Marriage and Family, 63 (1), 63-79.

Trickett, J. (2009). Community Psychology: Individuals and Interventions in Community Context. Annual Review of Psychology, 6. 395-419.

Vieno, A., Nation, M., Perkins, D. D. y Santinello, M. (2007). Civic participation and the development of adolescent behavior problems. Journal of Community Psychology, 35 (6), 761-777.

World Helth Organization. (2012). Risk behaviours: Being bullied and bullying others. En C. Currie, C. Zanaotti, A. Morgan, D. Currie, M. de Looze, C. Roberts, O. Samdal, O. R. F. Smith y V. Barnekow (Eds.), Social determinants of health and well-being among young people. Health Behaviour in School-aged Children(HBSC) study: International report from the 2009/2010 survey (pp. 191-200). Copenhagen: WHO Regional Office for Europe (Health Policy for Childrenand Adolescents, No. 6). Recuperado de http://www.euro.who.int/ data/assets/pdf file/0003/163857/Social -determinants-of-health-and-well-being-among-young-people.pdf.

(Articulo recibido: 8-10-2012; revisión recibida: 6-11-2012; aceptado: 3-7-2013) 